\title{
DOUBLY-PERIODIC SOLUTIONS OF A FORCED SEMILINEAR WAVE EQUATION
}

\author{
M. ARIAS, P. MARTÍNEZ-AMORES AND R. ORTEGA
}

(Communicated by Walter Littman)

\begin{abstract}
Existence results are obtained for doubly-periodic solutions of a semilinear wave equation when the nonlinearity is bounded in one side.
\end{abstract}

In this work we study the existence of weak doubly $2 \pi$-periodic solutions of the semilinear wave equation

$$
u_{t t}-u_{x x}+g(u)=f(t, x), \quad(t, x) \in R^{2},
$$

where $f$ is a given $2 \pi$-periodic function in $t$ and $x, g$ is a continuous function, and we assume, among other conditions to be specified later, the following:

$$
g \text { is nondecreasing and } g(-\infty)>-\infty, \quad g(+\infty)=+\infty
$$

When one looks for periodic spatially homogeneous (i.e. independent of $x$ ) solutions of (1), then one is lead to the periodic problem for the O.D.E.

$$
\frac{d^{2} u}{d t^{2}}+g(u)=f(t)
$$

It is known that this last problem admits a solution when $g$ satisfies (2) and $(1 / 2 \pi) \int_{0}^{2 \pi} f>g(-\infty)$. (See for example $[5,7]$.) The motivation of this paper is to extend in a certain sense this result to equation (1).

The Dirichlet-periodic boundary value problem for (1) has been extensively studied by Bahri and Brezis [1] (see also [2, 3]). Their corresponding condition on $g$ is

$$
g \text { is nondecreasing and }|g(u)| \leq \gamma|u|+c, \quad u \in R,
$$

where $\gamma$ and $c$ are constants with $\gamma<\left|\lambda_{-1}\right|$. Here $\lambda_{-1}$ is the first negative eigenvalue of the linear operator $\square=\partial^{2} / \partial t^{2}-\partial^{2} / \partial x^{2}$ when it acts on functions sastisfying the boundary conditions. Hypothesis (3) does not allow the crossing of $g$ and the eigenvalues of $\square$ different from $\lambda_{0}=0$. Also, it is obvious that that the growth of $g$ must be of linear type at most. The results in [1] can be easily translated to the doubly-periodic case.

In contrast with (3), our condition on $g$ is of a different nature. Actually, when (2) is verified, $g$ may cross other eigenvalues besides $\lambda_{0}$ or grow arbitrarily in the

Received by the editors July 18, 1986.

1980 Mathematics Subject Classification (1985 Revision). Primary 35L70, 35L05.

Key words and phrases. Nonlinear wave equation, doubly periodic solutions, resonance, $L^{\infty}$. bounds.

The authors wish to acknowledge the support from CAICYT, Ministerio de Educación y Ciencia, Spain. 
positive direction. On the other side $g$ must be bounded in the negative axis, which is not required by (3).

We should also mention the paper of Ward [8] on the doubly-periodic problem for (1). The results in [8] are related to ours, although $g$ is not allowed to interact with $\lambda_{0}$.

Further discussions on the connections with those works will be given at the end of the paper.

Our method of proof is based on a simple idea. We consider a sequence of truncated equations such that it is possible to apply the results in [1] to each one of them. Then we obtain a uniform $L^{\infty}$-bound on the solutions of the corresponding truncated problems; implying therefore that the solution of some of these problems is, at the same time, a solution of the original one. The technique used to get the bounds is based on [1] combined with some additional estimates similar to those in [8].

Preliminaries. Denote by $H$ the Hilbert space $L^{2}(J), J=(0,2 \pi) \times(0,2 \pi)$, with inner product $(\cdot, \cdot)$. Throughout the paper, a function on $J$ will be identified, whenever needed, to its doubly-periodic extension to $R^{2}$.

The realization in $H$ of the wave operator with periodic conditions, denoted by $A$, is defined as follows. Let $D$ be the class of test functions $\varphi \in C^{2}(\bar{J})$ verifying

$$
\begin{gathered}
\varphi(t, 0)-\varphi(t, 2 \pi)=\varphi_{x}(t, 0)-\varphi_{x}(t, 2 \pi)=0, \\
\varphi(0, x)-\varphi(2 \pi, x)=\varphi_{t}(0, x)-\varphi_{t}(2 \pi, x)=0, \quad t, x \in[0,2 \pi] .
\end{gathered}
$$

$A$ is given by$$
D(A)=\left\{u \in H / \varphi \in D \rightarrow \int_{J} u \square \varphi \text { is } L^{2} \text {-continuous }\right\},
$$$$
(u, \square \varphi)=(A u, \varphi) \quad \text { for every } u \in D(A), \quad \varphi \in D .
$$

It is known that $A$ is a selfadjoint unbounded linear operator in $H$ with closed range and $\lambda_{0}=0$ is an eigenvalue of $A$ of infinite multiplicity. The kernel and the range of $A$ are explicitly given by

$$
\begin{aligned}
& N(A)=\left\{u_{0} \in H / u_{0}(t, x)=\bar{u}_{0}+p(t+x)+q(t-x) \text { a.e. } J\right. \\
& \left.\bar{u}_{0} \in R ; p, q \in L_{\mathrm{loc}}^{2}(R), 2 \pi \text {-periodic and } \int_{0}^{2 \pi} p=\int_{0}^{2 \pi} q=0\right\} \\
& \begin{array}{r}
R(A)=N(A)^{\perp}=\left\{u_{1} \in H / \int_{0}^{2 \pi} u_{1}(t-x, x) d x\right. \\
\left.\quad=\int_{0}^{2 \pi} u_{1}(t+x, x) d x=0, \text { a.e. } t \in(0,2 \pi)\right\}
\end{array}
\end{aligned}
$$

and the natural projection onto the kernel,

$$
P u(t, x)=\bar{u}+p(t+x)+q(t-x), \quad u \in H,
$$

where

$$
\begin{gathered}
\bar{u}=\frac{1}{(2 \pi)^{2}} \int_{J} u, p(t)=\frac{1}{2 \pi} \int_{0}^{2 \pi} u(t-x, x) d x-\bar{u} \\
q(t)=\frac{1}{2 \pi} \int_{0}^{2 \pi} u(t+x, x) d x-\bar{u}
\end{gathered}
$$


We shall need the following regularity result (see [4]). Given $f \in R(A)$, let $u \in R(A)$ be the unique solution of $A u=f$ in $R(A)$. Then

$$
u \in L^{\infty}(J), \quad\|u\|_{L^{\infty}} \leq c\|f\|_{L_{1}}
$$

for some constant $c$ independent of $u$ and $f$.

By a weak solution of the doubly $2 \pi$-periodic problem or (1) we understand a function $u \in L^{\infty}(J)$ such that

$$
(u, \square \varphi)+(g(u), \varphi)=(f, \varphi) \text { for every } \varphi \in D .
$$

Clearly, $u \in L^{\infty}(J)$ verified (6) if and only if $u$ verifies

$$
A u+g(u)=f \text {. }
$$

We can now state our result.

THEOREM. Assume that $g$ satisfies (2) and the following condition is verified:

$$
\begin{aligned}
& f \in L^{\infty}(J) \text { admits a decomposition in the form } f=f^{*}+f^{* *} \text { with } \\
& f^{*} \in R(A) \cap L^{\infty}(J), f^{* *}(t, x) \geq g(-\infty)+\delta \text { a.e. }(t, x) \in J \text { for some }
\end{aligned}
$$

$\delta>0$. Then there exists at least one solution of $(7)$ in $L^{\infty}(J)$.

In the proof of the theorem we shall need a preliminary result that can be proved following the lines of [1].

LEMMA. Assume

(i) $g$ is a bounded nondecreasing continuous function.

(ii) $f \in L^{\infty}(J)$ admits a decomposition in the form $f=f^{*}+f^{* *}$ with $f^{*} \in$ $R(A), g(+\infty)-\delta \geq f^{* *}(t, x) \geq g(-\infty)+\delta$ a.e. $(t, x) \in J$ for some $\delta>0$. Then there exists at least one solution of $(7)$ in $L^{\infty}(J)$.

PROOF OF THE THEOREM. It is not restrictive to assume $g(-\infty)=0$. In consequence $g \geq 0$ over the entire real line. Let us consider the sequence of truncated functions

$$
g_{n}(u)=\min [g(u), g(n)], \quad n=1,2, \ldots,
$$

and the corresponding problems

$$
A u+g_{n}(u)=f .
$$

It is clear that $g_{n}$ verifies condition (i) of the previous lemma and, since $g_{n}(-\infty)=$ $g(-\infty)=0$, and $g_{n}(+\infty)=g(n)$, (ii) is verified for $n$ sufficiently large. Hence, for large $n,(9)$ admits a solution $u_{n} \in L^{\infty}(J)$. We will conclude the proof by showing the existence of an $L^{\infty}$-estimate of $u_{n}$ independent of $n$, implying therefore that $g_{n}\left(u_{n}\right)=g\left(u_{n}\right)$ and $u_{n}$ is a solution of (7) for large $n$.

We denote by $C_{i}, i=1,2, \ldots$, positive constants independent of $n$. Each $u_{n}$ can be decomposed as $u_{n}=u_{0 n}+u_{1 n}$ with $u_{0 n}=P u_{n}, u_{1 n}=(I-P) u_{n}$.

Since constant functions belong to $N(A)$ and $g_{n}\left(u_{n}\right)-f \in R(A)$, one has $\left(g_{n}\left(u_{n}\right)-f, 1\right)=0$. Now,

$$
\left\|g_{n}\left(u_{n}\right)\right\|_{L^{1}}=\int_{J} g_{n}\left(u_{n}\right)=\int_{J} f \quad\left(g_{n} \geq 0\right)
$$

and

$$
\left\|A u_{n}\right\|_{L^{1}}=\left\|g_{n}\left(u_{n}\right)-f\right\|_{L^{1}} \leq \int_{J} f+\|f\|_{L^{1}}
$$


From (5) we obtain

$$
\left\|u_{1 n}\right\|_{L^{\infty}} \leq C_{1} .
$$

By (2) and (8) one can find positive constants $\gamma$ and $k$ such that

$$
\left[g_{n}(u+\xi)-f^{* *}(t, x)\right] u \geq \gamma|u|-k
$$

for $u \in R,|\xi| \leq C_{1},(t, x) \in J$, and large $n$.

Using the facts that $u_{0 n} \in N(A)$ and $g_{n}\left(u_{n}\right)-f^{* *} \in R(A)$, and applying (11) with $u=u_{0 n}, \xi=u_{1 n}$, one gets

$$
\gamma \int_{J}\left|u_{0 n}\right|-(2 \pi)^{2} k \leq\left(g_{n}\left(u_{0 n}+u_{1 n}\right)-f^{* *}, u_{0 n}\right)=0
$$

from where

$$
\left\|u_{0 n}\right\|_{L^{1}} \leq C_{2}
$$

We can now write $u_{0 n}$ as

$$
u_{0 n}(t, x)=\bar{u}_{0 n}+p_{n}(t+x)+q_{n}(t-x)
$$

with $p_{n}, q_{n}$ essentially bounded, $2 \pi$-periodic and with mean value zero. The relations (4) together with (12) imply

$$
\left|\bar{u}_{0 n}\right|,\left\|p_{n}\right\|_{L^{1}},\left\|q_{n}\right\|_{L^{1}} \leq C_{3} .
$$

Therefore it is enough to find $L^{\infty}$-estimates for $p_{n}$ and $q_{n}$.

Since $g_{n}\left(u_{n}\right)-f^{* *} \in R(A)$,

$$
\begin{aligned}
& \int_{0}^{2 \pi} g_{n}\left(u_{n}(t-x, x)\right) d x=\int_{0}^{2 \pi} f^{* *}(t-x, x) d x \\
& \int_{0}^{2 \pi} g_{n}\left(u_{n}(t+x, x)\right) d x=\int_{0}^{2 \pi} f^{* *}(t+x, x) d x
\end{aligned}
$$

a.e. $t \in(0,2 \pi)$. From the previous estimates,

$$
u_{n}(t, x) \geq-C_{4}+p_{n}(t+x)+q_{n} /(t-x) \text { a.e. } J, C_{4}=C_{1}+C_{3},
$$

and (14) together with the monotonicity of $g_{n}$ imply

$$
\int_{0}^{2 \pi} g_{n}\left(-C_{4}+p_{n}(t)+q_{n}(x)\right) d x \leq 2 \pi\left\|f^{* *}\right\|_{L^{\infty}} .
$$

Let $M_{n}=\operatorname{ess} \sup _{(0,2 \pi)} p_{n}\left(M_{n} \geq 0\right.$ because $\left.\int_{0}^{2 \pi} p_{n}=0\right)$ and

$$
\Sigma_{n}=\left\{x \in(0,2 \pi):\left|q_{n}(x)\right| \geq M_{n} / 2\right\} .
$$

By (13) $\operatorname{meas}\left(\Sigma_{n}\right) \leq 2 C_{3} / M_{n}$, and by (16)

$$
\begin{aligned}
2 \pi\left\|f^{* *}\right\|_{L^{\infty}} & \geq \int_{\Sigma_{n}} g_{n}+\int_{[0,2 \pi]-\Sigma_{n}} g_{n} \\
& \geq g_{n}\left(-C_{4}+p_{n}(t)-\frac{M_{n}}{2}\right) \cdot\left(2 \pi-\frac{2 C_{3}}{M_{n}}\right) \quad \text { a.e. } t \in(0,2 \pi) .
\end{aligned}
$$


It follows

$$
\min \left\{g(n), g\left(-C_{4}+M_{n} / 2\right)\right\}\left(2 \pi-2 C_{3} / M_{n}\right) \leq 2 \pi\left\|f^{* *}\right\|_{L^{\infty}},
$$

which forces the boundedness of $M_{n}$.

Changing the roles of $p_{n}$ and $q_{n}$, starting with (15), and repeating the process one also find upper bounds for $q_{n}$. Say $p_{n}(t), q_{n}(t) \leq C_{5}$ a.e. $t$. Going back to (14) and using the estimate

$$
u_{n}(t, x) \leq C_{4}+p_{n}(t+x)+C_{5}
$$

one obtains

$$
g_{n}\left(C_{4}+C_{5}+p_{n}(t)\right) \geq \delta>0 \text { a.e. } t .
$$

Let $N_{n}=\operatorname{essinf}_{(0,2 \pi)} p_{n}\left(N_{n} \leq 0\right)$. Then

$$
g_{n}\left(C_{4}+C_{5}+N_{n}\right) \geq \delta>0
$$

which implies the boundedness of $N_{n}$, since $g(-\infty)=0$. A similar reasoning for $q_{n}$ using (15) finishes the proof.

REMARKS. 1. Hypothesis (2) of the theorem can be generalized in the following sense: $g$ is monotone and exactly one of the limits $g(+\infty), g(-\infty)$ is finite. (Of course (8) may need a slight change.)

2. Hypothesis (8) was first formulated in $[\mathbf{1}, \mathbf{2}]$ and, as mentioned there in a similar context, is a sharp condition for the solvability of (7). In fact, when $g(-\infty)<g(u)$ for all $u \in R$ (and this is the case for an increasing $g$ ) it is easily seen that (8) characterizes the solvability of (7). However, when $g(-\infty)=g(u)$, $u \leq c$ for some $c,(8)$ is only sufficient. A necessary condition for the solvability is

$$
\begin{aligned}
& f \in L^{\infty}(J) \text { admits a decomposition in the form } f^{*}+f^{* *} \text { with } \\
& f^{*} \in R(A) \cap L^{\infty}(J), f^{* *}(t, x) \geq g(-\infty) \text { a.e. }(t, x) \in J .
\end{aligned}
$$

We do not know whether this last condition is also sufficient in this case or not.

3. Some model nonlinearities verifying (2) are $\alpha u^{+}(\alpha \leq 1), u^{2} u^{+}, e^{u}, \ldots$ The results in $[1,8]$ do not apply to these examples. On the other hand we cannot study a nonlinear term of the type $\alpha u^{+}-\beta u^{-}(0<\alpha, \beta<1)$ that can be studied from the results in $[\mathbf{1}$ or $\mathbf{8}]$.

4. A key factor in our proof is the existence of positive functions in the kernel of $A$. Therefore it is not possible to adapt the proof to a Dirichlet-periodic problem (DP) for equation (1) of the type studied in [1]. Assuming that the (DP) problem is posed over $(0,2 \pi) \times(0, \pi)$ the function $\phi_{1}(t, x)=\sin x$ belongs to $N\left(A-\lambda_{1} I\right), \lambda_{1}=$ 1. It seems possible, however, to obtain some results when the resonance is at $\lambda_{1}$ by using the positivity of $\phi_{1}$.

5. Apparently, the obtaining of bounds of the solution relies very heavily on the specific structure of $N(A), A=\square$. It would be of interest to obtain results of a similar flavor for other semilinear problems with an infinite-dimensional kernel. A good example might be the problem induced by the beam equation. A representation of $N(A), A=\partial^{2} / \partial t^{2}+\partial^{4} / \partial x^{4}$, may be seen in [6].

\section{REFERENCES}

1. A. Bahri and H. Brezis, Periodic solution of a nonlinear wave equations, Proc. Roy. Soc. Edinburgh Sect. A 85 (1980), 313-320.

2. H. Brezis, Periodic solutions of nonlinear vibrating strings and duality principles, Bull. Amer. Math. Soc. (N.S) 8 (1983), 409-426. 
3. H. Brezis and L. Nirenberg, Forced vibrations for a nonlinear wave equation, Comm. Pure Appl. Math. 31 (1978), 1-30.

4. S.-N. Chow and J. Hale, Methods of bifurcation theory, Springer-Verlag, Berlin and New York, 1982.

5. R. Kannan and R. Ortega, Periodic solution of pendulum-type equations, J. Differential Equation 59 (1985), 123-144.

6. N. Krylová and O. Vejvoda, A linear and weakly nonlinear equation of a beam: The boundaryvalue problem for free extremities and its periodic solution, Czechoslovak Math. J. 21(96) (1971), 535-566.

7. J. Ward, Asumptotic conditions for periodic solutions of ordinary differential equations, Proc. Amer. Math. Soc. 81 (1981), 415-420.

8. $\_$, A wave equation with a possibly jumping nonlinearity, Proc. Amer. Math. Soc. 92 (1984), 209-214.

DEPARTAMENTO ECUACIONES FUnCIONALES, UNIVERSIDAD DE GRANADA, 18071 GRANADA, SPAIN 